\title{
Sistematización de una estrategia de educación informal implementada en personas privadas de la libertad en el establecimiento penitenciario de mediana seguridad y carcelario de Barranquilla, Colombia
}

\author{
Rafael Humberto Herrera-Mercado \\ Trabajador Social \\ Corporación Universitaria Rafael Núñez. Barranquilla, Colombia \\ https://orcid.org/0000-0001-8661-5572・rherreram26@curnvirtual.edu.co \\ Rafael Alberto Zambrano-Vanegas \\ Magíster en Gobierno y Políticas Públicas. Trabajador Social \\ Corporación Universitaria Rafael Núñez. Barranquilla, Colombia \\ https:/ / orcid.org/0000-0002-5609-2712・rafael.zambrano@curnvirtual.edu.co
}

\section{Resumen}

En este artículo se habla de un proceso de intervención sobre la comunidad de personas privadas de la libertad (PPL) del Establecimiento Penitenciario de Mediana Seguridad y Carcelario de Barranquilla (EPMSCBA-ERE) que por su calidad de sindicados no tenían acceso a la redención de pena. Se elaboró una sistematización de experiencia por un equipo interdisciplinario, quienes definieron los ejes de sistematización, reconstruyeron la experiencia, e hicieron la reflexión crítica, donde identificaron aspectos que permitieron construir e implementar un proyecto de Educación Informal. Para ello se trabajó por medio de una encuesta, grupos focales y talleres lúdicos reuniones de trabajo, y revisión de todo el sustento documental de la información registrada de la experiencia. La sistematización permitió a todos los actores del proceso reconocer que debe existir una responsabilidad de todas las partes, para reconocer nuevas vías de llegar a la readaptación de las PPL. Se llegó a la conclusión de que es necesario comprender la importancia que tiene la educación como herramienta resocializadora para las personas privadas de la libertad así como acentuar el rol que ejerce el Trabajador Social y otros profesionales afines. Por otro lado, se logró que los participantes del programa se visibilizaran y asumieran como "sujetos de derechos", y fueran parte activa del proceso.

Palabras clave: Educación; Persona privada de la libertad; Resocialización; Sistematización; Tratamiento penitenciario.

Recibido: 10/08/2020 |Aprobado: 21/11/2020 | Publicado: 01/01/2021

(i)(2) Esta obra está bajo una Licencia Creative Commons Atribución-NoComercial-CompartirIgual 4.0 Internacional.

Financiación o proveniencia del artículo: artículo derivado del proyecto de Sistematización de una experiencia de implementación de una estrategia de educación no formal para personas privadas de la libertad en el Establecimiento Penitenciario de Mediana Seguridad y Carcelaria de Barranquilla, realizado en el Instituto Nacional Penitenciario y Carcelario INPEC, avalado por la Corporación Universitaria Rafael Núñez campus Barranquilla, Colombia.

¿Cómo citar este artículo? / How to quote this article?

Herrera-Mercado, R. H., y Zambrano-Vanegas, R. A. (2021). Sistematización de una estrategia de educación informal implementada en personas privadas de la libertad en el establecimiento penitenciario de mediana seguridad y carcelario de Barranquilla, Colombia. Prospectiva. Revista de Trabajo Social e intervención social, (31), 241-257. doi: 10.25100/prts.v0i31.10491. 
Herrera-Mercado y Zambrano-Vanegas

\title{
Systematization of a Strategy of Informal Education Implemented with Inmates of a Medium Security Penitentiary Establishment in Barranquilla, Colombia
}

\begin{abstract}
This article talks about a process of intervention in a community of people deprived of their liberty (PDL) in a medium security penitentiary establishment in Barranquilla (Establecimiento Penitenciario de Mediana Seguridad y Carcelario de Barranquilla EPMSCBA-ERE). The target population did not have access to reduction of sentence. A systematization of the experience was elaborated by an interdisciplinary team who defined the axes of the systematization, reconstructed the experience, critically reflected about the process and identified aspects that allowed for the construction and implementation of an informal educational project. Data was collected through a survey, focus groups, playful workshops, work meetings and a review of all the documentary support of the information recorded about the experience. The systematization permitted all the stakeholders to recognize their share of the responsibility and identify new avenues to arrive at the readaptation of the PPL/PDL. It was concluded that it is necessary to comprehend the importance of education as a resocializing tool for PPL/PDL, and the role of the Social Worker and other related professionals in this process. The process also achieved visibility for the participants and that they participated actively and viewed themselves as "subjects with rights".
\end{abstract}

Keywords: Education; Person deprived of liberty; Resocialization; Systematization; Prison treatment.

Sumario: 1. Introducción, 2. Metodología, 3. Hallazgos, 3.1. Discusión, 3.2 Los puntos de llegada, 4. Conclusiones, 5. Referencias Bibliográficas. 


\section{Introducción}

La sistematización de la experiencia de implementación del proyecto de educación informal tuvo como propósito lograr que la comunidad de personas privadas de la libertad (PPL), ubicadas en el Establecimiento Penitenciario de Mediana Seguridad y Carcelario (EPMSCBA-ERE) de Barranquilla, Colombia, en calidad de sindicados, reconocieran y trabajaran el significado e importancia que tiene el Modelo Educativo Institucional del INPEC (Instituto Nacional Penitenciario y Carcelario) como parte de su intervención en el contexto penitenciario, en la promoción y garantía del derecho a la redención de penas, en el marco del desarrollo del proceso de resocialización promovido por este modelo educativo institucional, orientado a facilitar la integración social de los sindicados.

El tratamiento penitenciario es una estrategia pedagógico-terapéutica para reinsertar en la sociedad a los individuos condenados por infringir la ley. El propósito de este es potencializar las capacidades y habilidades del individuo por medio del trabajo, el estudio y la enseñanza. Desde esta perspectiva, la educación es entendida como uno de los pilares fundamentales del ser humano, se convierte en una herramienta importante de la intervención de "tratamiento penitenciario" y derecho a la redención de penas en los centros carcelarios y penitenciarios del país. Por tal razón, reducir los impactos negativos, asociados a la ausencia de educación formal e informal y a las condiciones sociales que promueven comportamientos de reincidencia de la población privada de la libertad es un tema prioritario.

De esta manera, se consideró pertinente diseñar un proyecto de educación informal para la institución y para todos sus actores, es en esta instancia donde se establece una caracterización de las necesidades de formación y de participación de las personas privadas de la libertad en condición de sindicados, para generar soluciones creativas, con participación colectiva de las personas privadas de la libertad del establecimiento.

Por otro lado, es de vital importancia generar conciencia a partir de la labor de formación, teniendo en cuenta que las limitaciones de cobertura, recursos pedagógicos disponibles e infraestructura educativa son debilidades y amenazas que retratan las actuales realidades sociales de las PPL.

El proyecto se desarrolla en tres etapas. La primera se centra específicamente en la población sindicada, que por causa de su proceso no puede acceder a los planes de educación formal. Se contó con una participación de veinte (20) personas privadas de la libertad en condición jurídica de sindicados, de una población estimada de 900 personas sindicadas presentes y 751 personas en condición jurídica de condenadas, ubicadas en una infraestructura física diseñada y construida para albergar un máximo de 600 personas privadas de la libertad, con un total de 1.651 personas privadas de la libertad presentes en 
Herrera-Mercado y Zambrano-Vanegas

el establecimiento penitenciario de mediana seguridad de Barranquilla. Para las personas sindicadas están estipulados unos planes de atención en los cuales se busca caracterizar a los futuros participantes y darles una formación que debe realizarse con la población interna dentro de los patios del establecimiento penitenciario.

La segunda etapa contempló el desarrollo de un proyecto educativo dirigido a las personas privadas de la libertad, mediante una estrategia de formación y capacitación en temas como valores humanos, derechos humanos, deportes y recreación dirigida, literatura, relaciones interpersonales, emprendimiento y labores industriales (panadería, artesanía), todo esto basado en transferencia y apoyo; con técnicas participativas en relación con la convivencia, el modelo educativo, las prácticas de intervención y el tratamiento de las personas privadas de la libertad en su ámbito psicosocial.

La tercera corresponde a una etapa de implementación para establecer mecanismos de concertación y coordinación para que el diseño del proyecto educativo informal pueda llegar en el futuro a la mayor cantidad de personas privadas de la libertad como sea posible.

En síntesis, este proyecto pretende recuperar, analizar y organizar los lineamientos concernientes al proceso educativo informal del establecimiento penitenciario. De esta manera, este puede ser replanteado en sus bases, diseño y formulación institucional.

Al desarrollar el proyecto, se tuvo como propósito la sistematización y reconstrucción de las líneas de investigación y los procesos generadores de conocimiento, por parte de los Trabajadores Sociales, al tiempo que apuntó a proveer al proyecto educativo informal dirigido a las personas privadas de la libertad (PPL) una estrategia metodológica para conformar redes de saberes, con las cuales se pudieran desarrollar plataformas de información y conocimiento con la comunidad penitenciaria y administrativa. De allí, el proyecto desarrolló sus potencialidades para generar beneficios entre los usuarios y participantes del proceso de resocialización.

\section{Metodología}

El proceso de sistematización se desarrolló desde el macrosistema hasta su microsistema, donde las características psicológicas de las personas privadas de libertad así como sus aptitudes, permitieran desarrollar acciones a partir de las necesidades de estos y del personal del establecimiento penitenciario y carcelario; esto con el fin de permitir triangular la información, incorporar las experiencias de todos los actores involucrados en él y considerar todos los niveles de impacto donde incidir para garantizar avances en el proyecto educativo de educación informal. 
Para todo el proceso metodológico se tuvieron en cuenta los pasos para sistematización desarrollados por Jara-Holliday (2018): el punto de partida, las preguntas iniciales, la recuperación del proceso vivido, la reflexión de fondo: ¿por qué pasó lo que pasó? y los puntos de llegada.

La sistematización tuvo un carácter participativo en el que se llevaron a cabo reuniones de trabajo, 20 encuestas de caracterización, 20 entrevistas individuales, 2 grupos focales y revisión del material de información registrado a través de la experiencia, desarrollados en fases y etapas. A continuación, se presentan las fases y etapas del proyecto educativo:

\section{Fases del Proyecto Educativo}

1. Presentación del Proyecto. Presentación Proyecto "Edúcate para la Libertad".

2. Sensibilización de los actores del proyecto. Las Personas Privadas de la Libertad se sensibilizan de la importancia de la estrategia educacional como mecanismo de apoyo en la redención de pena.

3. Aplicación de la instrucción metódica. Educación Informal dentro de los patios del EPMSCBA- ERE para que tenga la capacidad de modificar su comportamiento social.

4. Implementación de los Talleres. Direccionar a las Personas Privadas de la Libertad para que tenga la capacidad de transformar su comportamiento social y generar de su parte su proyecto de emprendimiento.

\section{Etapas del Proyecto Educativo}

1. Presentación del Proyecto

2. Caracterización de las Personas Privadas de la Libertad

3. Sensibilización de las Personas Privadas de la Libertad

4. Aplicación de la Estrategia de Educación Informal

5. Seguimiento y Monitoreo

6. Sistematización de la Experiencia 


\section{Hallazgos}

\subsection{El punto de partida}

Durante todo el proceso inicial y hasta la sistematización se recogió información relevante alrededor de la situación de las PPL, quienes evidenciaban en sus comentarios hacia los funcionarios la necesidad que tenían de querer realizar alguna actividad que les permitiera redimir su pena. Debido a estas sugerencias y necesidades del contexto nació este proyecto como una propuesta de solución a esta problemática.

El contexto de la educación informal implementado en el Sistema Penitenciario y Carcelario en Colombia, está reglamentado por la Ley General de la Educación (1994), cuyo artículo 68 define el objetivo y ámbito de los programas de rehabilitación social para aquellas personas que necesitan reincorporarse a la sociedad, además el artículo 69 establece que este tipo de educación requiere métodos didácticos, contenidos y procesos pedagógicos acordes con el contexto y sus educandos (Español-Sierra y Moreno-Farías, 2014), donde los establecimientos penitenciarios implementan los planes y programas educativos y orientaciones pedagógicas administradas por el Instituto Nacional Penitenciario y Carcelario, INPEC.

Ante el escenario descrito anteriormente, lo primero que se analizó fue la norma contemplada en la Resolución 3190 de 2013, “Por la cual se determinan y reglamentan los programas de trabajo, estudio y enseñanza válidos para evaluación y certificación de tiempo para la redención de penas en el Sistema Penitenciario y Carcelario administrado por el Instituto Nacional Penitenciario y Carcelario, INPEC".

A través de este análisis se discutió sobre los diferentes aspectos relevantes como la dignidad de las PPL, la resocialización, los tipos de educación y las metodologías a utilizar; entendiendo que estas cuestiones estaban interrelacionadas y apuntaban en la misma dirección. Frente a la resocialización, esta se define desde una postura positivista y funcionalista por el Sistema Penitenciario y Carcelario como "tratamiento penitenciario" que se propone examinar la personalidad del infractor de la ley penal, a través de la disciplina, el trabajo, el estudio, la formación espiritual, la cultural, el deporte y la recreación, bajo un espíritu humano y solidario (Ley 65, 1993).

En el contexto institucional penitenciario y carcelario de Colombia, el INPEC (2016) ha definido la resocialización como la técnica de tratamiento clínico que pretende cambiar la conducta del interno (a). Resocializarse, implica aprender las expectativas sociales e interiorizar normas de conducta para el desarrollo de habilidades sociales. Resocializarse es volver a valer como ser social conforme quiere la sociedad (p. 11). 
A su vez, la Corte Constitucional de Colombia define la resocialización como un derecho al que deben tener acceso todos los individuos privados de la libertad en establecimientos carcelarios en Colombia, mediante la Sentencia T-267 de 2015, que plantea el derecho a participar en los programas de educación formal y trabajo con el objetivo de preparar a las personas privadas de la libertad en condición jurídica de condenados para su vida en libertad.

Sin embargo, este derecho de resocialización definido por el Sistema Penitenciario y Carcelario no se cumple plenamente para las personas privadas de la libertad en condición de sindicados, ya que por su situación transitoria que presume de su inocencia, donde no se ha definido su status jurídico al encontrarse en proceso de juicio (si se absuelve o se condena), no logran obtener este beneficio de la educación formal, por la temporalidad y particularidad de cada caso de las personas sindicadas, que dependen de las respuestas y dinámicas propias de la Administración de Justicia, siendo los sindicados una población carcelaria en condición de vulnerabilidad que no son objeto directo de los programas de educación formal, ya que pueden permanecer durante 4 o más años en condición de sindicado sin definirse su situación jurídica, lo que agrava su permanencia en los establecimientos penitenciarios, caracterizados por el hacinamiento y las consecuentes condiciones de insalubridad y de conflictividad.

Después de haber comprendido cómo se llegó a decidir sobre el desarrollo de este proyecto educativo dirigido a las PPL sindicadas y teniendo en cuenta los ejes de sistematización que se habían propuesto como eran: la participación de las PPL, el desarrollo del proceso de Resocialización, la estrategia de Educación Informal para PPL sindicados y la apropiación y proyección del proceso de resocialización en las PPL, se dispuso la formulación de un proyecto desde el enfoque de marco lógico formativo que estableciera el cronograma y su forma de trabajo, especialmente para las PPL en condición de sindicados. A continuación se muestra en la Tabla 1 el marco lógico formativo. 
Tabla 1. Marco lógico formativo.

Impacto general del proyecto: implementar estrategias comunes que contribuyan hacia el efectivo desarrollo del derecho a la educación de las personas privadas de la libertad y al mejoramiento de sus oportunidades de inclusión social y redención de pena.

Objetivo del Proyecto: implementar una Estrategia de Educación Informal para Personas Privadas de la Libertad en el EPMSCBA - ERE.

\section{Resultado esperado 1}

Se ha caracterizado a las PPL de manera que se distingan claramente los beneficiarios del proyecto.

\section{Actividades}

Presentación proyecto "Edúcate para la libertad".

Encuestas de caracterización de las PPL.

Grupos focales de estudio de las opiniones o actitudes de PPL.

Resultado esperado 2

Actividades

Exposición de las ventajas de la educación como herramienta de cambio.

Talleres lúdicos para acercar a la realidad a las PPL

Las PPL se sensibilizan de la importancia de la estrategia educacional como mecanismo de apoyo en la redención de pena.

de la necesidad de realizar una actividad como la educación.

Dinámicas de sensibilización por medio de actividad didáctica (juegos) donde se sensibiliza a las PPL y se eliminan barreras que lo predisponen a no educarse.
Resultado esperado 3

Taller nueva vida: por medio de la aplicación de la instrucción metódica, se busca direccionar a las PPL, para que tenga la capacidad de modificar su comportamiento por medio del crecimiento intelectual y espiritual que fortalezcan valores, el cumplimiento de sus metas, emprendimiento y la toma decisiones.

\section{Resultado esperado 4}

La aplicación de la estrategia de Educación Informal dentro de los patios del EPMSCBA-ERE permite a la mayor cantidad de personas privadas de la libertad acceder a la redención de pena.

Actividades
Módulo Fortaleza, con base en el yo de cada
persona se divide en tres partes: el yo, los demás y
el ambiente social.
Módulo Objetivo, el cual se divide en: mi tesoro,
mis ambiciones y mi realidad.
Módulo Emprendedor. Se divide en: confío en mí,
los demás confían, el ambiente confía, confío en
Dios.
Modulo Decisión se divide en: lo mío, de ustedes,
de todos.
Diseño de la estrategia de talleres de literatura,
deportes, arte, promoción y prevención en salud y
psicosociales con fines de tratamiento
penitenciario.
Implementación de los talleres de literatura,
deportes, arte, promoción y prevención en salud y
psicosociales con fines de tratamiento penitenciario
Seguimiento y monitoreo.
Sistematización de la experiencia.

Fuente: elaboración propia 
Herrera-Mercado y Zambrano-Vanegas

Para los funcionarios públicos es difícil recibir las quejas de la población en general sobre la entidad en la que trabaja, ya que eso genera una necesidad de proteger a la institución; sobre todo una que viene siendo desprestigiada, tanto por la situación carcelaria en Colombia y sus causales como hacinamiento, problemas de salubridad, salud, etc., además de problemas de reincidencia de la población privada de la libertad, hasta los debates de siempre sobre corrupción y malos manejos de los presupuestos.

Se puede pensar que, bajo una situación tan precaria, puede carecer de importancia el proceso de resocialización de las Personas Privadas de la Libertad (PPL) sindicadas, un proceso que a su vez es cuestionado por su eficacia en la forma en que está implementándose dentro de estos establecimientos penitenciarios en la actualidad.

Sin embargo, se hace necesario proveer al funcionario de estrategias metodológicas que le permitan aportar a su conocimiento para revertir la situación y que a su vez esta pueda mejorar. Es por esto que, a partir de la formación profesional en Trabajo Social de los autores y la oportunidad que se presentaba de aprovechar las prácticas profesionales de grado de uno de los autores, se tuvo la idea de atender las necesidades educativas existentes dentro del establecimiento penitenciario, la cual era la de implementar con el proceso de resocialización a las comunidades privadas de la libertad en calidad de sindicada.

Este factor fundamental llevó a proponerle a la Junta de Evaluación de Trabajo, Estudio y Enseñanza (JETEE) del establecimiento penitenciario que, teniendo en cuenta la Resolución 3190 del 2013, mencionada anteriormente, se implementara un Proyecto Educativo Informal que se constituyera en un posible modelo de atención en educación informal desarrollado por la institución, coherente con otras estrategias pedagógicas articuladas al proceso de resocialización que ya existía en el establecimiento penitenciario de Barranquilla, orientado a la población privada de la libertad en condición de sindicados, que se encontraban sin ninguna actividad de redención ni de productividad.

Una vez puesto en conocimiento de la JETEE la problemática de esta población sindicada, se decidió que un proyecto de educación informal sería la mejor opción para acceder a la población en los patios; teniendo en cuenta las restricciones de seguridad y de aislamiento que se tienen dentro del establecimiento penitenciario.

De ahí que al plantearse los ejes sobre los que giraría el proyecto, se permitiría tener la participación activa de las PPL, así como el desarrollo del proceso de Resocialización y éste, una vez avanzado, se tendría la apropiación y proyección para el desarrollo del mismo en las PPL de este plan piloto, como una política institucional dentro del establecimiento penitenciario, buscando que las posibilidades de que las personas sindicadas puedan acceder a un trabajo y a adaptarse nuevamente a la sociedad. 
Herrera-Mercado y Zambrano-Vanegas

El proyecto de educación informal dirigido a las PPL sindicadas es una estrategia que sirve para la generación de estrategias en materia de garantía de derechos de todo tipo para las PPL, además que, parte de un cambio de orientación del proceso de intervención en la resocialización a la población sindicada y puede ser un avance de los presupuestos de la educación como instrumento para promover un cambio de modelo pedagógico articulado al modelo terapéutico que se implementa actualmente.

El INPEC ya contaba con el Plan de Acción y Sistema de Oportunidades (P.A.S.O) (Ramírez-López, Vélez-Gutiérrez y Díaz-Galvis, 2004) el cual fue implementado en el año 2002. Este constituye una propuesta de planeación, organización y ejecución del tratamiento, cuya virtud es recopilar lo ordenado en el Código Penitenciario y Carcelario (Ley 65, 1993).

Este sistema cobijaba a las PPL en condición de sindicados, quienes desarrollaban actividades de formación en artesanías, pero la gran mayoría no realizaban estas actividades por falta de material, razón por la cual el proyecto de educación informal se orientaba a implementar las herramientas establecidas en el P.A.S.O, adaptándose a las circunstancias particulares de las PPL sindicadas que por su condición jurídica, no solo no estaban inscritos en ninguna actividad de redención o estaban inscritos sin realizar una actividad real, sino que además estaban desperdiciando su tiempo en privación de la libertad.

Uno de los factores que se tuvieron en cuenta a la hora de articular el proyecto fue el Modelo Sistémico y Ecológico del mismo, entendiendo que este:

\begin{abstract}
Pasa del estudio del individuo aislado al estudio del sistema y las relaciones entre sus elementos que lo conforman (...) Desde el punto de vista sistémico es muy importante trabajar atendiendo, en la medida de lo posible, a todos los sistemas involucrados en la vida de la persona. La prioridad está en la intervención en el sistema más cercano y estable en la vida del individuo y este acostumbra a ser el familiar. (El modelo de intervención sistémico nos insta a evaluar y trabajar de manera global, pensando que la "causa" de los problemas no es la persona aislada o, por lo menos, entender que los factores de mantenimiento de un problema se encuentran en las relaciones del sistema y no en su origen). (AnimaTEA, 2015)
\end{abstract}

De esta manera, se buscó que, por medio de un conjunto de actividades educativas, instructivas, laborales y de aprendizaje sobre cómo recuperar y mantener sus relaciones familiares, con una influencia en su entorno social, se lograra promover la formación de nuevos proyectos de vida en las PPL sindicadas, para su reintegración a la libertad, desde una mirada Sistémica y Ecológica, para prevenir la reincidencia desde los contextos de ilegalidad.

Comenzar el proyecto de educación informal para las PPL sindicadas no fue tarea fácil, había que presentar y explicar el proyecto a sus beneficiarios, buscando capturar su 
interés y así lograr que se inscribieran como candidatos, ya que los cupos para el plan piloto, fueron estipulados por la dirección del establecimiento penitenciario en 30, para el manejo operativo y logístico en el contexto penitenciario.

Seguido a esto se aplicaron 20 encuestas y se organizaron 2 grupos focales, con el fin de tomar en cuenta las necesidades del grupo, lo cual contribuyó al diseño del plan de acción y comenzar la etapa de selección de los candidatos, quienes una vez aceptados, firmaron un acta de compromiso que, a pesar de las dificultades propias del medio penitenciario, participarían en el proceso de educación informal hasta su finalización, o hasta la obtención de su libertad (según cada caso), teniendo en cuenta su condición de sindicados.

La población participante del proceso de educación informal tuvo en cuenta la oferta que ya establecía el establecimiento penitenciario y carcelario de Barranquilla, la cual está basada en cuatro elementos: cobertura, continuidad, pertinencia y logros.

Se evidenciaron obstáculos relacionados con su continuidad en el proceso de educación informal, esto debido a que los sindicados por su estatus jurídico son una población transitoria dentro del establecimiento penitenciario, ya que una vez terminado el proceso judicial, o es liberado al ser exonerado de los cargos, o es trasladado en la mayoría de los casos al haber sido dictada la pena condenatoria, así que la persona privada de la libertad, si es condenada, debe comenzar un nuevo proceso, dentro del P.A.S.O. Esta situación planteó un reto en cuanto al modelo y proceso de educación en condiciones de calidad para ofrecer a grupos de estudiantes que inicien el proceso, pero que posiblemente no lleguen a terminarlo.

Se gestionó con el Servicio Nacional de Aprendizaje SENA, la implementación de la modalidad de educación informal, para el desarrollo de cursos del nivel técnico (ver Tabla 2); de esta manera, se ofreció a las PPL sindicadas una estructura pedagógica orientada hacia la formación en derechos humanos y el desarrollo de habilidades sociales orientadas hacia el desarrollo de emprendimientos. Los cursos que se seleccionaron para el proyecto de educación informal fueron:

Tabla 2. Cursos cortos del SENA.

\begin{tabular}{ll}
\hline Curso & Duración \\
\hline Básico en emprendimiento & 40 horas \\
Proyecto de vida & 40 horas \\
Mercadeo y ventas & 40 horas \\
Higiene y manipulación de alimentos & 40 horas \\
Elaboración de alimentos de panificación de & 40 horas \\
manera artesanal en panadería & \\
\hline
\end{tabular}

Fuente: elaboración propia 
Herrera-Mercado y Zambrano-Vanegas

Los cursos del SENA aportaron desde su implementación al cumplimiento misional de la institución penitenciaria y carcelaria en el proceso de resocialización, orientada a la formación de proyectos personales de vida que, en condiciones de libertad o de privación de la misma, aportan a la formación y capacitación a personas, que evidencian carencias en niveles de escolaridad y de formación para el trabajo.

En el cumplimiento de los propósitos del proyecto de educación informal para PPL sindicadas, se gestionó ante el establecimiento penitenciario, su apoyo en la caracterización y selección de la población en condición de sindicados, a partir de las características diversas de los grupos poblacionales existentes (indígena, raizal, afro, LGTBI), y de sus necesidades de resocialización.

Teniendo en cuenta que la resocialización debe ser considerada un derecho subjetivo, en el cual la PPL sindicadas tienen una situación jurídica en particular, se constituyen como titulares de derechos subjetivos. Al ser considerada la resocialización como un derecho fundamental, la intervención del Estado, a través del INPEC y de los organismos de control (Ministerio Público), debe garantizar su implementación con coherencia y responsabilidad social, ante la ausencia de programas institucionales de educación formal dirigidos a las personas privadas de la libertad sindicadas, predominan posturas políticas e institucionales que rechazan la viabilidad y el impacto social de los procesos de resocialización y limitan este proceso a acciones aisladas, de carácter asistencialista, sin coordinación ni coherencia ni claridad pedagógica.

La ausencia de programas y proyectos de educación formal e informal dirigidos a las PPL sindicadas generan problemas de ansiedad, depresión, baja autoestima, confusión, afectando su salud mental en medio de la privación de su libertad y con percepciones de abandono social y estatal, que conduce a una "victimización", que impide su participación en acciones de resocialización y por ende, considera que al terminar su privación de la libertad va a ser despreciado por la sociedad que lo odia por el error que cometió. Ese odio que cree que la sociedad le tiene, se convierte en el odio que seguramente le llevará a delinquir nuevamente, bajo el precepto de "venganza" que arrastra su pensamiento. Esta situación nace como lo expresa Carrasco-Jiménez (2007), en su artículo El pensamiento penal de Michel Foucault:

Como el delincuente, se situaba por el delito fuera de la ley, se dejaba caer sobre él la venganza de este soberano. Así, con el castigo, se trata de establecer una "simetría de la venganza" (Idem), donde se iguala lo horrendo del delito con un castigo igualmente horrendo. De ahí la crueldad de los castigos. Así el suplicio era la manifestación del castigo, y el objeto, el cuerpo del delincuente, estableciéndose una relación íntima entre éste y aquel. El dolor que significa la imposición del castigo, está directamente dirigido al cuerpo. El verdugo, representa así y por ende, la realidad de dicho castigo. (p. 3) 
Herrera-Mercado y Zambrano-Vanegas

Es un círculo vicioso de venganza de la sociedad hacia el infractor y de este hacia la sociedad. Ahí radica la importancia de la implementación de proyectos de educación formal e informal hacia la comunidad privada de la libertad sindicada, programas y proyectos culturales, políticos, económicos, incluso espirituales, además de la gestión de políticas públicas orientadas hacia la resocialización, que les permita acciones de perdón al infractor y de integración en las actividades económicas, sociales y culturales en la cotidianidad.

Uno de los primeros desafíos del proyecto educativo informal implementado con las personas privadas de la libertad sindicadas fue el de sensibilizarlas y formarlas en derechos humanos, para transformar imaginarios negativos y aportar a la recuperación de su autoestima, de sus vínculos familiares y de prevención del consumo de sustancias psicoactivas, propias del contexto penitenciario.

Las condiciones institucionales y ambientales del establecimiento penitenciario, expresadas en los controles de seguridad para neutralizar los levantamientos de la población carcelaria en general, la negligencia del personal de custodia para realizar el traslado al lugar de enseñanza y los problemas de escasez de espacios físicos y la poca disponibilidad de horarios y de espacios reducidos e improvisados, determinaron el desarrollo de la experiencia de implementación del proyecto de educación informal en el establecimiento penitenciario y carcelario de Barranquilla, que plantearon la necesidad de implementar actividades culturales y de desarrollo integral en temas del orden psicosocial relacionados con hábitos, normas, actitudes y prejuicios.

Fue necesario entender que el INPEC bajo sus premisas de seguridad y de limitación de recursos institucionales, desempeña un rol elemental de apoyo, pero insuficiente en su esfuerzo institucional para implementar acciones de resocialización a las PPL sindicadas, donde es necesaria la intervención de equipos interdisciplinarios compuestos por Trabajadores Sociales, Psicólogos, Educadores y auxiliares ocupacionales que direccionen los programas y proyectos de educación y de resocialización.

\subsection{Los Puntos de llegada}

La sistematización de experiencias es "producto del esfuerzo por construir marcos propios de interpretación teórica desde las condiciones particulares de nuestra realidad" (Jara-Holliday, 2018, p. 27). La sistematización de la experiencia desarrollada en el establecimiento penitenciario, siguiendo sus objetivos y ejes de sistematización, permitió que la participación de las PPL tuviera un reconocimiento interno en el establecimiento de Barranquilla, entrando en una relación recíproca entre las PPL sindicadas y los funcionarios públicos del INPEC, reconociendo, legitimando y validando los derechos que tiene esta comunidad de personas sindicadas a la dignidad, la educación y la reintegración a la sociedad. 
Otro punto de llegada en la sistematización de la experiencia de desarrollo del proyecto de educación informal fueron los aprendizajes significativos para todos los actores sociales involucrados, en especial la promoción y experiencia de participación social de las personas privadas de la libertad sindicadas, en diferentes eventos, talleres, encuentros y sesiones que facilitaron el aprendizaje de los distintos temas, donde se evidenciaron avances en el estado de ánimo favorable al proceso formativo, en la motivación por participar y aportar en cada uno de los cursos y sesiones desarrolladas con la posibilidad de implementarlos en su futuro laboral y social.

Esto último fue crucial para lograr otro de los objetivos como era la apropiación y proyección del proceso de resocialización en las PPL sindicadas, con la intención de promover un ambiente favorable para un aprendizaje significativo que lograra enriquecer a estas con los conocimientos y experiencias que fortalecieran su autoestima y su proyecto personal de vida.

Se puede reflexionar sobre la experiencia vivida en el proceso de desarrollo del proyecto de educación informal para personas privadas de la libertad en el establecimiento penitenciario y carcelario de Barranquilla, como un paso necesario que debe dar el INPEC hacia la búsqueda de las mejores condiciones de vida para las PPL sindicadas, porque más allá del error cometido, merecen una nueva oportunidad de rehacer sus vidas, evitando con esto su reincidencia; la sistematización de la experiencia ha permitido recuperar, visibilizar y validar las vivencias de esta colectividad, permitiendo un diálogo entre las necesidades jurídicas y educativas de estos y la realidad de su vivencia diaria en condiciones de privación de la libertad como las de las personas condenadas en establecimientos penitenciarios de Colombia.

Este proceso de sistematización es un reconocimiento a los procesos de intervención que se efectúan a diario en los establecimientos penales del país, que actúan de manera aislada del sistema penitenciario y carcelario, pero si lograran integrarse, sus estrategias de resocialización serían más efectivas.

Los aprendizajes de la sistematización de la experiencia de educación informal implementada en el establecimiento penitenciario permiten unas nuevas posibilidades a que más PPL sindicadas y los funcionarios públicos promuevan espacios de reflexión, alrededor del proceso de resocialización a través de estrategias de apoyo psicosocial, emocional, jurídico y de gestión empresarial orientadas hacia la reintegración a la vida social en libertad.

La sistematización ha generado un espacio para que todos los actores sociales del proceso tengan una mirada retrospectiva de la resocialización, reconociendo que tiene 
Herrera-Mercado y Zambrano-Vanegas

innumerables fallas y ante estas, la generación de estrategias y compromisos institucionales de todas las partes, que permitan orientar a procesos de integración social de las PPL sindicadas.

Los profesionales involucrados en el proceso de resocialización, son transformadores y mediadores en materia cultural, jurídica, educativa y psicosocial, que contribuyan a una transición jurídica paralela a una integración a la sociedad con una visión emancipadora, crítica y autónoma, siendo la sistematización de la experiencia de implementación del proyecto de educación informal, una oportunidad para todos los actores sociales participantes en vivenciar y en diseñar una acción política a partir de la experiencia vivida

\section{Conclusiones}

Como expresa Jara-Holliday (s.f): "Son punto de partida para nuevos aprendizajes, por lo que pueden ser inquietudes abiertas pues no se trata de conclusiones 'definitivas'" (p. 12).

En este caso, la sistematización de la experiencia vivida en el establecimiento penitenciario y carcelario de Barranquilla, permite entender la importancia que tiene la educación como estrategia para la resocialización para las personas privadas de la libertad sindicadas. Además, acentúa el rol que ejerce el Trabajador Social en su interacción profesional con esta población.

Por medio de la participación de las PPL sindicadas en el proyecto de educación informal, se logró que los participantes se visibilizaran y asumieran como "sujetos de derechos", donde ellos mismos fueron parte activa del proceso y eso les permitió reflexionar sobre sus realidades particulares y colectivas, a partir de su participación en las diferentes actividades lúdicas, clases, encuentros y talleres de emprendimiento.

Acerca del desarrollo del proceso de resocialización, la sistematización permitió obtener aprendizajes alrededor de los ejes y objetivos específicos que fueron trazados, que fue de utilidad para obtener nuevas formas y estrategias metodológicas formativas que demuestran que además de las estrategias ya existentes como el Plan de Acción y Sistema de Oportunidades (P.A.S.O), pueden implementarse acciones afirmativas que refuercen el proceso en general, de acuerdo a las condiciones sociales y culturales de las personas privadas de la libertad sindicadas.

La reflexión acerca de la Estrategia de Educación Informal para PPL sindicadas, parte de una experiencia que permitió reafirmar el poder que tiene la educación como emancipadora de la mentalidad criminal, así como descubrir técnicas que se realizaron en la práctica, que influyeron en las PPL sindicadas participantes del proyecto y que 
Herrera-Mercado y Zambrano-Vanegas

evidenciaron que las estrategias pedagógicas implementadas fueron pertinentes y permitieron alcanzar en cierta medida, los objetivos enumerados en el proyecto.

Hay que insistir que el sistema penitenciario y el proceso de resocialización reflejan dificultades que parecen imposibles de superar, a causa del hacinamiento, fallas en los procesos de atención en salud y en temas de convivencia entre la comunidad de PPL, por eso, llevar a cabo la tarea de reintegrar al seno de la comunidad a quienes previamente han cometido delitos, requiere estrategias de apoyo individual y grupal que permita cambios de mentalidad y de actitud por parte de las PPL sindicadas; para ello la educación, la cultura, el deporte, la formación en emprendimientos y sobre todo la promoción de sus habilidades y capacidades sociales para que puedan, una vez en libertad, sostenerse económicamente ellos y sus familias, lo cual en gran medida les permitiría evitar la reincidencia.

Uno de los objetivos y ejes de sistematización de mayor dificultad, fue el de la apropiación y proyección del proceso de resocialización en las PPL sindicadas, ya que todo el proceso formativo en derechos humanos, inteligencia emocional, responsabilidad social y libertad, convivencia y emprendimiento, fuera un primer paso para su nueva vida fuera del centro penitenciario.

Es una realidad que las PPL sindicadas en su mayoría no creen en el proceso de resocialización, esto debido a la limitada eficacia del sistema penitenciario, dado que este proceso es un reflejo de la dinámica de la administración de justicia presente en todo el sistema penitenciario del país, con las evidentes dificultades en materia de seguridad alimentaria, atención en salud y condiciones de hacinamiento presentes en los establecimientos penitenciarios del país; no se puede esperar de manera ingenua que el proceso de resocialización sea exitoso.

Sin embargo, entre la mayoría de los participantes en el proyecto de educación no formal se promovió un cambio de pensamiento positivo al evidenciar un mejoramiento en la autoestima personal y en la estabilidad emocional de las PPL sindicadas, manifestadas en su participación y motivación a continuar en las acciones del proyecto de educación informal, que apoyan un cambio de personalidad y, por ende, en sus progresos en las metas trazadas a nivel individual y grupal.

Una de las áreas del proyecto de educación informal que más motivó la participación de las PPL sindicadas, fue el proceso de formación en emprendimiento, que les brinda herramientas para pensar en un futuro donde ellos puedan generar ingresos para la satisfacción de sus necesidades básicas y de sostenimiento familiar, sin la inclusión de terceros que podrían discriminarlos por su pasado en prisión. Al perder ese miedo a la discriminación y estigmatización por parte de la sociedad, se les facilita integrarse socialmente a sus comunidades. 
La sistematización de la experiencia permite desarrollar procesos de educación informal y formal similares y, de tener continuidad a mediano y largo plazo, no solo dentro del establecimiento penitenciario y carcelario de Barranquilla, también en otros establecimientos penitenciarios de Colombia, con el fin de ampliar el escenario de interacción profesional del Trabajo Social a través de estrategias metodológicas que permitan incidir en los contextos y en las comunidades de personas privadas de la libertad sindicadas y en sus familias, disminuyendo sus niveles de vulnerabilidad social a través de la formación y de la preparación para afrontar la vida en libertad o en privación de la misma.

\section{Referencias bibliográficas}

AnimaTEA (11 de junio de 2015). El Modelo Sistémico (una visión general). [Mensaje en un Blog]. Recuperado de http:/ / www.animatea.cat/Blog_El_Modelo_Sistemico.html.

Carrasco-Jiménez, E. (2007). El pensamiento penal de Michel Foucault. POLIS. Revista Latinoamericana, (18), 1-15. Recuperado de https://journals.openedition.org/polis/ 4138.

Congreso de Colombia. (19 de agosto de 1993). Código Penitenciario y Carcelario [Ley 65 de 1993]. DO: 40.999.

Congreso de Colombia. (8 de febrero de 1994). [Ley 115 de 1994]. Recuperado de https://www.mineducacion.gov.co/1621/articles-85906_archivo_pdf.pdf

Corte Constitucional de Colombia. (8 de mayo de 2015). Sentencia T-267 de 2015. Recuperado de https://www.corteconstitucional.gov.co/relatoria/2015/T-26715.htm.

Español-Sierra, W., y Moreno-Farías, B. (2014). La educación en cárceles, una experiencia desde un establecimiento de reclusión. Hojas y Tablas, (11), 7-19.

Instituto Nacional Penitenciario y Carcelario. (2016). Glosario penitenciario y Carcelario. Bogotá: INPEC.

Instituto Nacional Penitenciario y Carcelario. (23 de octubre de 2013). Resolución 3190.

Recuperado

http://epn.gov.co/elearning/distinguidos/TRATAMIENTO/1_resolucin_3190_del _23102013.html.

Jara-Holliday, O. (2018). La sistematización de experiencias: práctica y teoría para otros mundos posibles. Bogotá: Centro Internacional de Educación y Desarrollo Humano. CINDE.

Jara-Holliday, O. (s.f.). Orientaciones teórico-prácticas para la sistematización de experiencias. Recuperado de http://centroderecursos.alboan.org/ebooks/0000/0788/6_JAR_ORI.pdf.

Ramírez-López, H., Vélez-Gutiérrez, L., y Díaz-Galvis, M. (2004). Plan de acción y sistema de oportunidades P.A.S.O: Una estrategia para el tratamiento penitenciario. Instituto nacional penitenciario y carcelario. Bogotá: Instituto Nacional Penitenciario y Carcelario-INPEC. 


\section{OTROS ARTÍCULOS DE PROSPECTIVA No. 31 DE 2021}

\section{PRESENTACIÓN}

Presentación. Reflexiones sobre desafios al publicar sistematizaciones

Rosa María Cifuentes-Gil

\section{EDITORIAL}

Reflexiones sobre Trabajo Social: aportes de la Sistematización

María Rocío Cifuentes-Patiño

\section{ARTÍCULOS}

Hacer lo que se sabe, pensar lo que se hace. La sistematización como modalidad investigativa Alfonso Torres-Carrillo

Aportes y desafios de la Sistematización de experiencias en el Trabajo Social y la extensión crítica. Apuntes y reflexiones desde la perspectiva de la Educación Popular

María Rosa Goldar

Valeria Chiavetta

La sistematización en Trabajo Social y la epistemología feminista del punto de vista. Diálogos sobre la producción de conocimiento sustentada en experiencias

Ruth Noemí Parola

María Florencia Linardelli

La Sistematización investigativa de las experiencias: del baile de los que sobran a la fiesta de los que faltan

María Belén Ortega-Senet

Sistematización y Trabajo Social en Chile. El largo y sinuoso camino

Patricia Lorena Castañeda-Meneses

Ana María Salamé-Coulon
Sentipensar la pandemia COVID-19 desde la sistematización de la experiencia en Trabajo Social: reflexiones del profesor Oscar Jara Holliday

Elia Sepúlveda-Hernández

La sistematización de experiencias, una investigación social cualitativa que potencia buenas prácticas de convivencia y gobierno. La experiencia de un conjunto residencial multifamiliar en Cali, Colombia Martha Lucia Echeverry-Velásquez Manuela Prada-Dávila

Construcción de subjetividades epistemológicaspolíticas de profesoras y profesores de Investigación social en una universidad privada y confesional en Bogotá

Giovanni Mora-Lemus

Sistematización de la experiencia Reconocimiento de los derechos humanos del adulto mayor en dos familias residentes en Cali y Valledupar (Colombia)

Lina María Cuello-Lacouture

Jimena del Pilar Jaramillo-Jaramillo

La memoria transformadora como estrategia de intervención profesional en los procesos de reconciliación social: comprensión a partir de mujeres campesinas, excombatientes y jóvenes en Manizales, Colombia

Yeimmy Stephania Corredor-Sotelo

Juliana Fuertes-Fuertes

Sistematización de una estrategia de educación informal implementada en personas privadas de la libertad en el establecimiento penitenciario de mediana seguridad y carcelario de Barranquilla, Colombia

Rafael Humberto Herrera-Mercado Rafael Alberto Zambrano-Vanegas 
Aportes significativos del proceso de intervención comunitaria con la Escuela Popular de Comunicación Alternativa Jaime Garzón de la ciudad de Cúcuta, Colombia

Carlos Lasso-Urbano

La sistematización de la intervención como metodología de investigación en Trabajo Social. Importancia práctica y teórica de la fase de recogida de datos en la intervención social según experiencia del Programa de Apoyo a las Familias en Zaragoza, España

Elisa Esteban-Carbonell

Nuria Del Olmo-Vicén

Papel de la sistematización de experiencias en los procesos de evaluación de intervenciones de salud pública en la Comuna Saludable por la Paz, Cali - Colombia

Jenny Faisury Peña-Varón

Paola Andrea Marín-Velásquez

Janeth Mosquera-Becerra

Experiencia de intervención social en hogares comunitarios integrales del barrio Alfonso Bonilla Aragón, Cali - Colombia

Julián Alexander Montaño-Cárdenas

Las políticas sociales y el gobierno de la "población indígena". Estrategias y regulaciones en el multiculturalismo chileno

Rodrigo Agustín Navarrete-Saavedra

Representaciones sociales sobre estilo de autoridad y tipos de interacción en cuidadores de residencias de protección infantil en Chile

Marcelo Gallegos-Fuentes
Carmen Gloria Jarpa-Arriagada

Reflexiones sobre inseguridad social y cuestiones penales. Una respuesta estratégica a partir de experiencias de cooperativismo con ex detenidos en Argentina

Analia Elizabeth Otero

Yael Yanina Barrera

Desarrollo y salud: la emergencia de un nuevo paradigma

Jesús María Sánchez-Ordóñez

Trabajo Social en ejercicio libre: la perspectiva profesional en España

Paula Frieiro-Padín

Tamara Fernández-Arias

Rubén González-Rodríguez

\section{RESEÑAS}

Social Work and the City: Urban Themes in 21stCentury Social Work

Felipe Saravia-Cortés

Respuestas del Trabajo Social ante emergencias sociales y problemáticas sociales complejas de México y España

Felipe Saravia-Cortés

El feminismo, el género y la profesionalización del trabajo social en Colombia (1936-2004)

Ambar Oriana Serna-Lombo

El puño invisible. Arte, revolución y un siglo de cambios culturales

Carlos Arturo Robledo-Marín

\section{PROSPECTIVA}

No. $31 \bullet$ ene.-jun. 2021

e-ISSN: 2389-993X • Universidad del Valle 